Supporting Information

\title{
Three-Dimensional Integration of Functional Oxides and Crystalline Silicon for Optical Neuromorphic Computing using Nanometer-scale Oxygen Scavenging Barriers
}

J. Elliott Ortmann ${ }^{1}$, Albina Y. Borisevich ${ }^{2}$, Sunah Kwon ${ }^{3}$, Agham Posadas ${ }^{1}$, Moon J. Kim², Alexander A. Demkov ${ }^{1, *}$

${ }^{1}$ Department of Physics, The University of Texas, Austin, Texas 78712, USA

${ }^{2}$ The Materials Science and Technology Division, Oak Ridge National Laboratory, Oak Ridge, Tennessee 37831, USA

${ }^{3}$ Department of Materials Science and Engineering, The University of Texas at Dallas, Richardson, Texas 75080, USA

*demkov@physics.utexas.edu 


\section{Supporting Note 1 - XPS Measurements of Deposited Silicon}

One of the fundamental challenges of depositing crystalline silicon on transition metal oxide (TMO) thin films is prohibiting the spontaneous oxidation and amorphization of the deposited silicon due to oxygen scavenging ${ }^{1-3}$. In our heterostructures, the deposition of silicon on a $\mathrm{LaAlO}_{3}$ (LAO) oxygen scavenging barrier prohibits the silicon from oxidizing, as illustrated by in situ XPS measurements of the Si $2 p$ core level (Figure S1). A small signature of oxidation is observed in the spectrum of the silicon deposited at high temperature, while the predominant signal originates from elemental silicon. All signatures of oxidation are absent from the spectrum of the silicon deposited at low temperature.

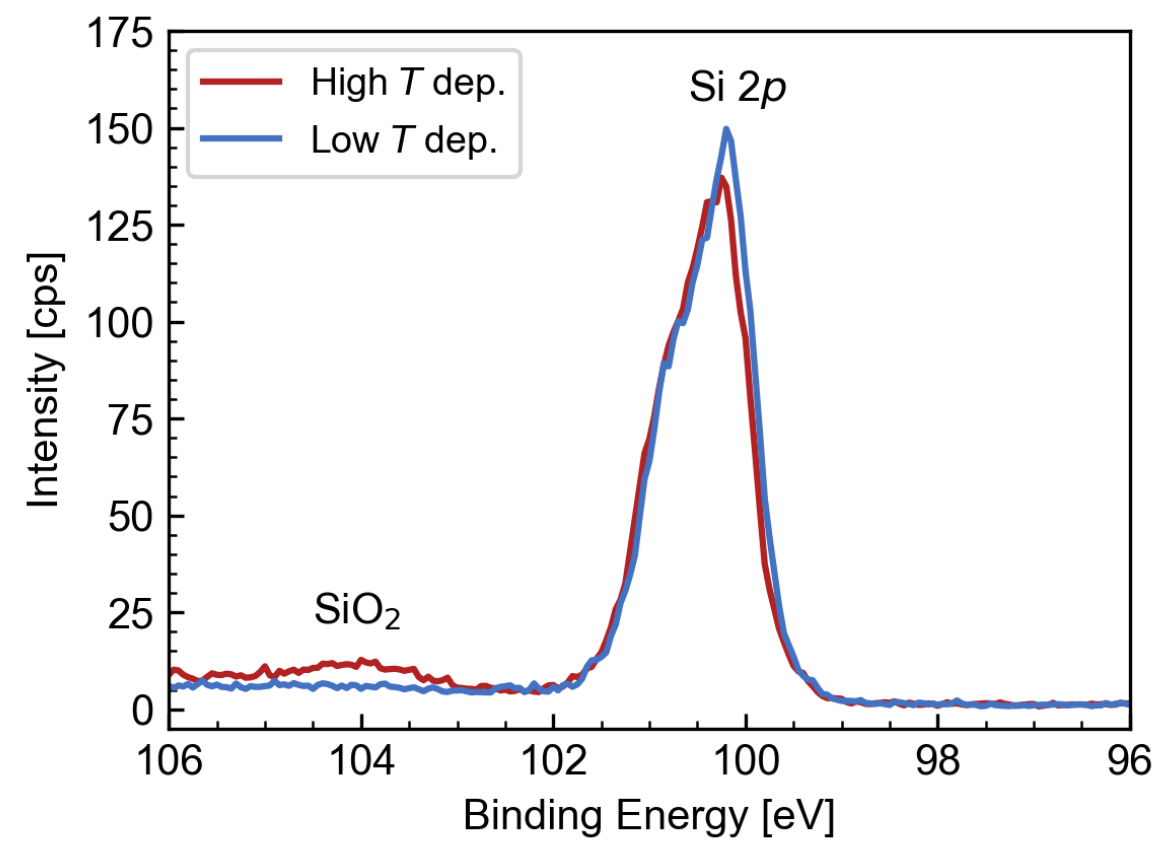

Figure S1. in situ XPS measurements of deposited silicon. Si $2 p$ core level spectra of silicon deposited on an LAO oxygen scavenging barrier. The silicon layer was first seeded at high temperature (red curve) followed by deposition at low temperature (blue curve). 


\section{Supporting Note 2 - EELS Mapping of Silicon/TMO/Silicon Superlattice}

Electron energy loss spectroscopy (EELS) mapping (Figure S2) was used to confirm the chemical assignment of each layer in the electron microscopy imaging (Figure 2 and 3, main text). Notably, the $\mathrm{O} K$ signal decays abruptly at both $\mathrm{Si} / \mathrm{TMO}$ interfaces (Figure S2b), supporting the conclusion from in situ XPS (Figure S1) that the deposited silicon does not scavenge oxygen from the underlying LAO oxygen scavenging barrier.
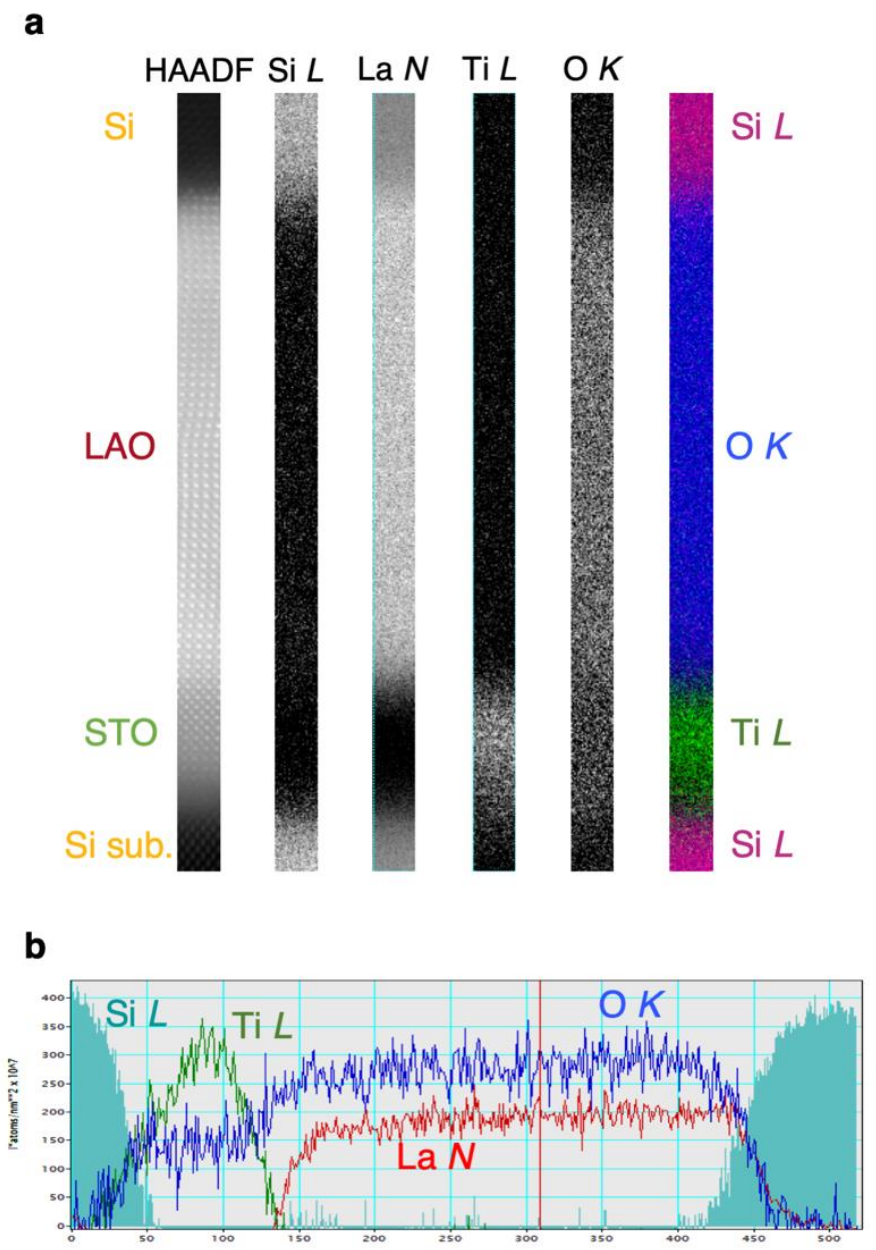

Figure S2. EELS mapping of heterostructure. (a) HAADF image of superlattice (left) compared with spatial mapping of Si $L$, La $N$, Ti $L$, and O $K$ EELS spectra. The combined EELS spectra are plotted in a spatial color map in the right-most image. (b) EELS spectra line scans through the heterostructure. 


\section{Supporting Note 3 - HAADF Images for GPA}

High-angle annular dark-field scanning transmission electron microscopy (HAADF STEM) images of the silicon/TMO/silicon superlattice (Figure S3) were collected simultaneously with the medium-angle annular dark-field (MAADF) STEM images presented in the main paper (Figure 2). The HAADF STEM images clearly demonstrate the epitaxial nature and high crystalline quality of the $\mathrm{SrTiO}_{3}$ (STO) and LAO thin films deposited on the silicon substrate. The high-resolution HAADF STEM image of the LAO/Si interface (Figure S3c) was utilized to perform geometric phase analysis (GPA) of the deposited silicon (Figure 3), revealing regions of large in-plane strain resulting from defects in the lattice of the deposited silicon.

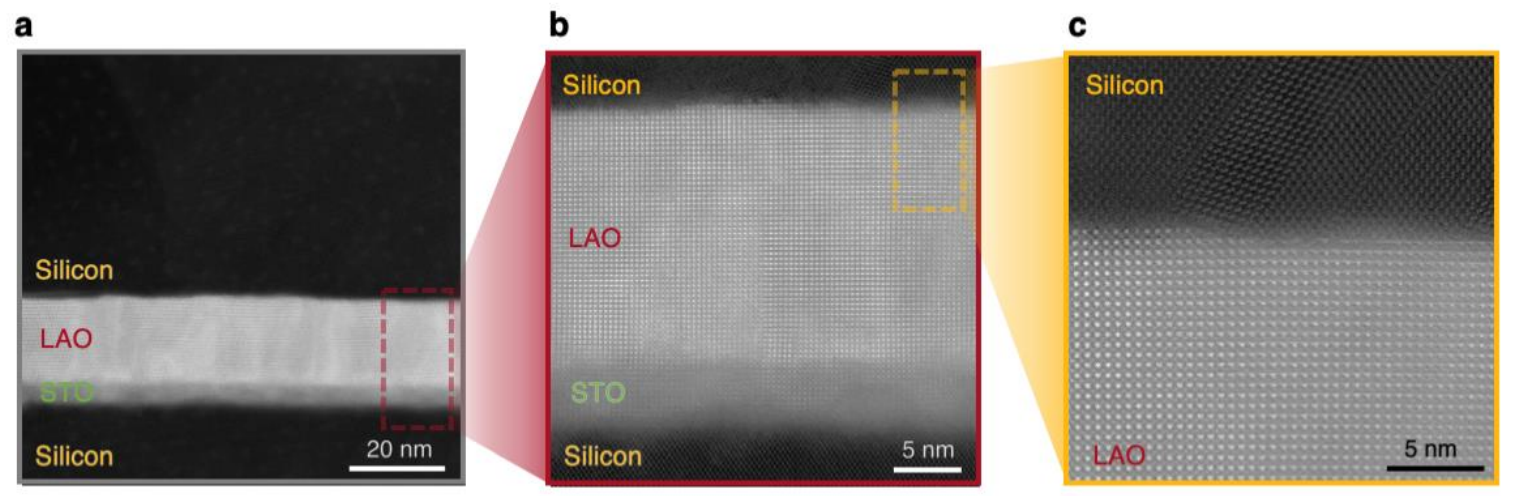

Figure S3. HAADF images of silicon/TMO/silicon heterostructure. Cross-sectional HAADF STEM images of (a) the silicon/TMO/silicon superlattice up to the deposited silicon layer (b) the TMO layer stack, and (c) the LAO/Si interface. 


\section{Supporting Note 4 -XRD Measurements of Silicon/TMO/Silicon}

\section{Superlattice}

$\mathrm{X}$-ray diffraction (XRD) measurements of a complete silicon/TMO/silicon superlattice (Figure S4) indicate the presence of predominantly (001)-oriented silicon. A small volume of the $\mathrm{Si}(111)$ phase is also present (Figure S4a,b), in agreement with the electron diffraction and electron microscopy results in the main text (Figure 1, 2, 3). The presence of the low-intensity, forbidden $\operatorname{Si}(002)$ peak is common in symmetric XRD spectra of high-quality silicon substrates ${ }^{4}$. As expected, the epitaxial TMO layers (STO and LAO) show only ( $00 l)$ orientation, where $l=1,2,3$. The splitting between the STO and LAO peaks can be seen clearly in the scan around the $\operatorname{TMO}(002)$ lattice peak (Figure S4c).
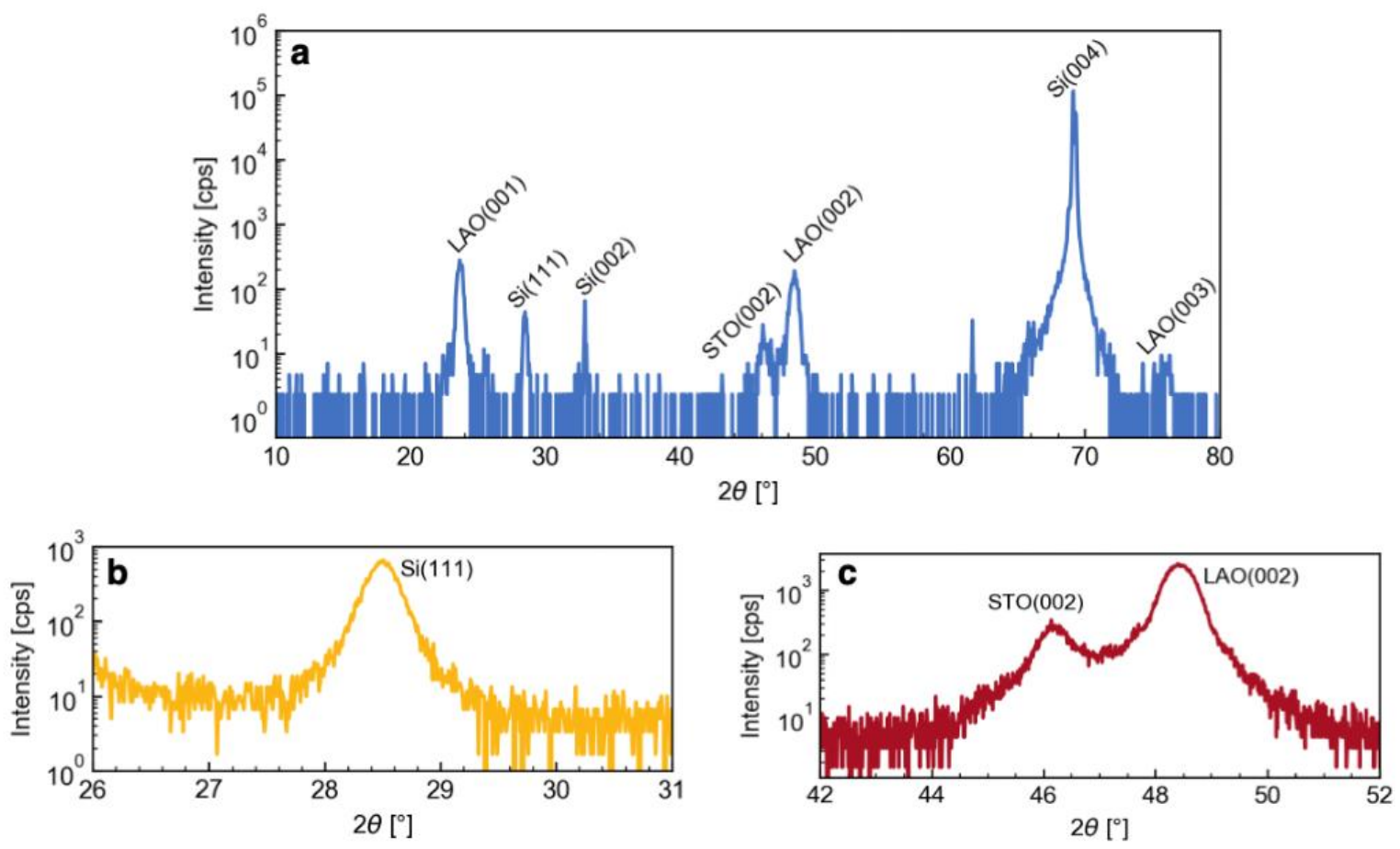

Figure S4. XRD of silicon/TMO/silicon heterostructure. Out-of-plane symmetric $\theta / 2 \theta$ XRD patterns of the full silicon/TMO/silicon superlattice, including the top epitaxial STO layer, showing (a) the survey spectrum (b) fine scan of the Si(111) peak, and (c) fine scan of the TMO(002) peaks (STO and LAO).

The XRD rocking curve of the Si(111) lattice peak (Figure S5) indicates the presence of significant mosaicity in the $\mathrm{Si}(111)$ grains of the deposited silicon. This finding is in agreement with the GPA results in the main text (Figure 3), which reveal defects at grain boundaries in the deposited silicon. Using a Pearson VII fit to the rocking curve data (solid line, Figure S5), a full width at half maximum (FWHM) $\delta \omega=1.69^{\circ}$ is extracted. 


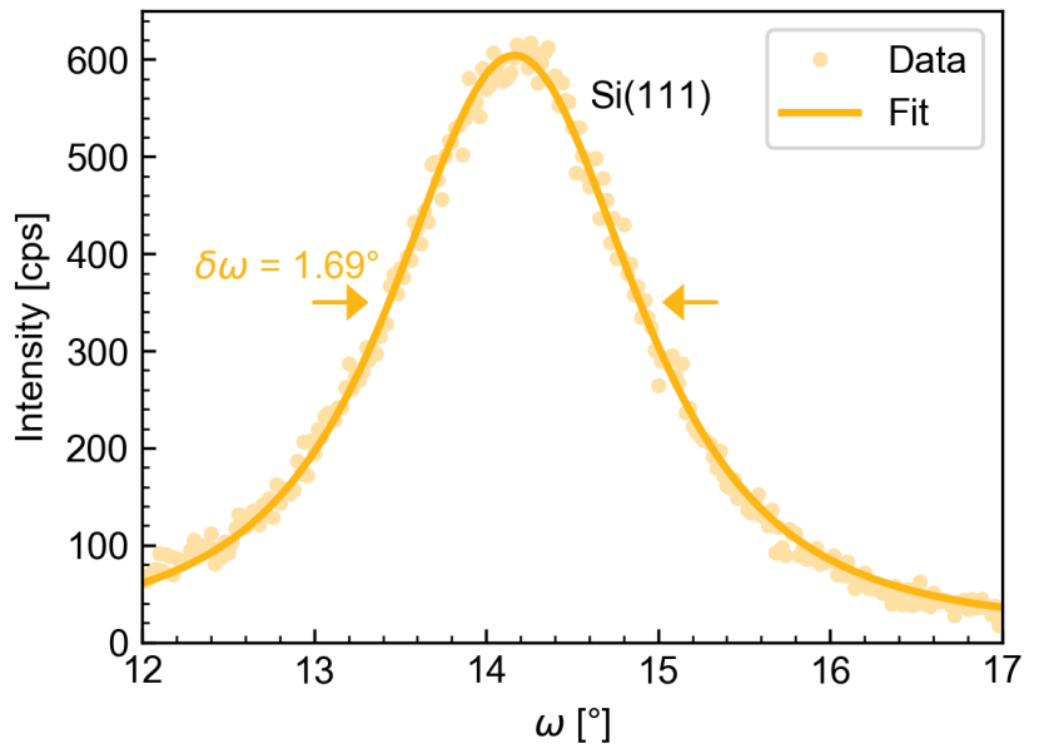

Figure S5. XRD rocking curve. Rocking curve ( $\omega$ scan) of the Si(111) lattice peak. Light yellow circles are data points while the solid yellow line is a Pearson VII fit to the data. A FWHM $\delta \omega=1.69^{\circ}$ is extracted from the fit. 


\section{Supporting Note 5 - Selected Area Electron Diffraction of Deposited Silicon}

While the XRD measurements and electron microscopy images of the silicon/TMO/silicon heterostructures demonstrate the presence of $\mathrm{Si}(111)$ domains in the deposited silicon, the RHEED pattern (Figure 1) and successful growth of epitaxial STO on the deposited silicon (Figure 1, 4) suggest that the deposited silicon primarily crystallizes in the $\mathrm{Si}(001)$ orientation. Indeed, selected area electron diffraction (SAED) measurements of the deposited silicon along two different zone axes show a majority $\mathrm{Si}(001)$ orientation of the deposited silicon film (Figure S6a, c). Comparison of the film SAED patterns with those taken from the silicon substrate (Figure S6b, d) reveals the presence of additional silicon orientations in the film besides $\mathrm{Si}(001)$ via the appearance of additional diffraction spots. However, the volume fraction of the additional silicon orientations appears to be small and likely decreases as the silicon film is grown thicker, as suggested by the results in the main text.
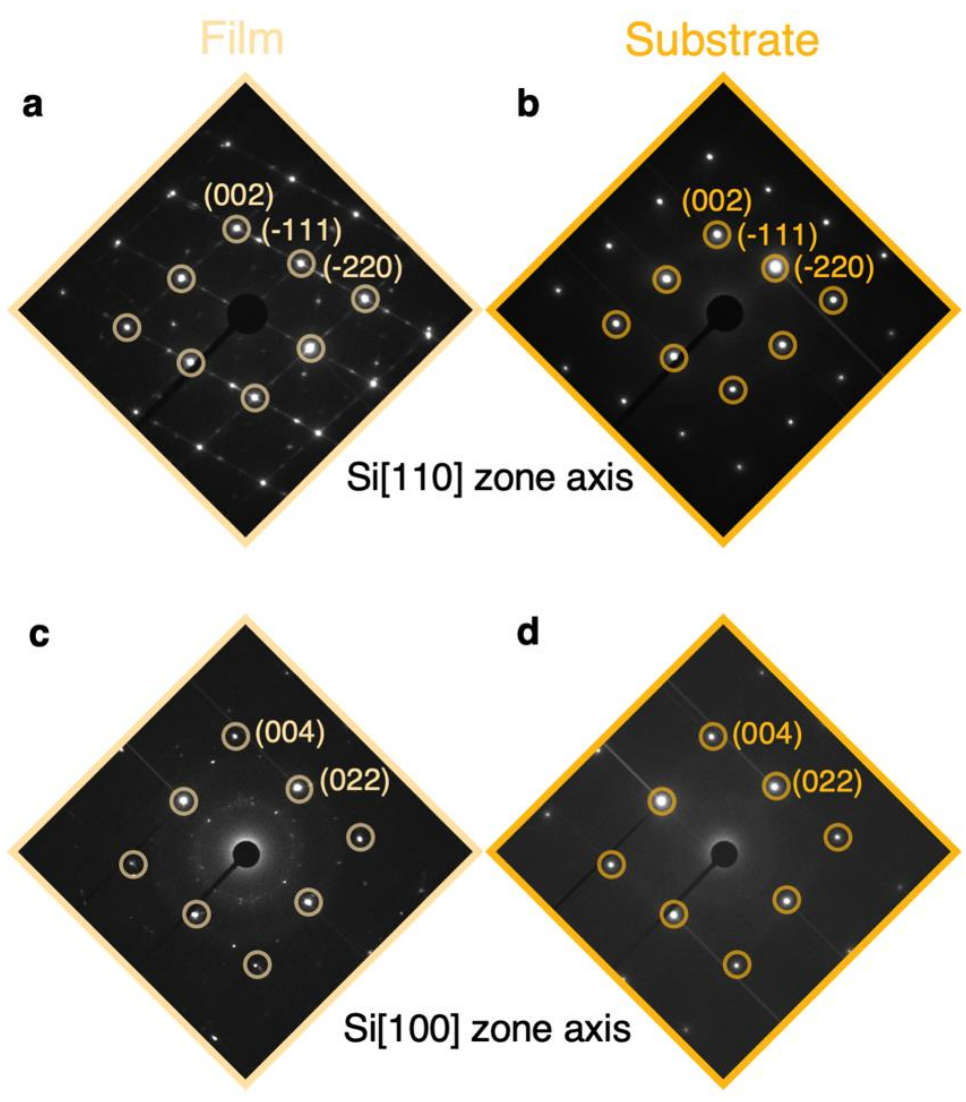

Figure S6. Selected area electron diffraction of silicon. SAED measurements of the (a) silicon film and (b) silicon substrate along the Si[110] zone axis. SAED measurements of the (c) film and (d) substrate along the Si[100] zone axis. Major silicon diffraction spots are marked by open circles in all four diffraction patterns. 


\section{References}

(1) Posadas, A. B.; Kormondy, K. J.; Guo, W.; Ponath, P.; Geler-Kremer, J.; Hadamek, T.; Demkov, A. A. Scavenging of Oxygen from $\mathrm{SrTiO}_{3}$ during Oxide Thin Film Deposition and the Formation of Interfacial 2DEGs. J. Appl. Phys. 2017, 121 (10), 105302. https://doi.org/10.1063/1.4978248.

(2) Guo, W.; Posadas, A. B.; Lu, S.; Smith, D. J.; Demkov, A. A. EuO Epitaxy by Oxygen Scavenging on $\mathrm{SrTiO}_{3}$ (001): Effect of $\mathrm{SrTiO}_{3}$ Thickness and Temperature. J. Appl. Phys. 2018, 124 (23), 235301. https://doi.org/10.1063/1.5059560.

(3) Kormondy, K. J.; Gao, L.; Li, X.; Lu, S.; Posadas, A. B.; Shen, S.; Tsoi, M.; McCartney, M. R.; Smith, D. J.; Zhou, J.; et al. Large Positive Linear Magnetoresistance in the TwoDimensional $t_{2 g}$ Electron Gas at the EuO/SrTiO 3 Interface. Sci. Rep. 2018, 8 (1), 7721. https://doi.org/10.1038/s41598-018-26017-z.

(4) Ortmann, J. E.; McCartney, M. R.; Posadas, A.; Smith, D. J.; Demkov, A. A. Epitaxial Oxides on Glass: A Platform for Integrated Oxide Devices. ACS Appl. Nano Mater. 2019, 2 (12), 7713-7718. https://doi.org/10.1021/acsanm.9b01778. 